\title{
Modern Vaccines- an Overview
}

\section{H A Bharat*}

Department of Veterinary Pathology, Karnataka Veterinary, Animal and Fisheries

Sciences University, Bidar, India

*Corresponding Author: H A Bharat, Department of Veterinary Pathology,

Karnataka Veterinary, Animal and Fisheries Sciences University, Bidar, India.
Received: November 25, 2021

Published: February 18, 2022

(C) All rights are reserved by $\mathbf{H} \mathbf{A}$ Bharat.

DOI: $10.31080 /$ ASVS.2022.04.0333

\begin{abstract}
Vaccines are one of the greatest inventions in modern medicine, vaccines have played a significant role in control, eradication of lethal diseases like smallpox and are responsible for the robust improvement in life expectancy over the past 2 centuries. Edward jenner pioneered the development of vaccinology by demonstrating immunity to smallpox when inouculated with cow pox scabs. The development of live attenuated vaccines and inactivated vaccines in $19^{\text {th }}$ played a major role in prevention of diseases like cholera, rabies, anthrax. Early $20^{\text {th }}$ century saw development of sub-unit vaccines which possessed only the antigenic part of microbe making it safer than live attenuated vaccines, with the advent of recombinant DNA technology and proteomics in the later decades of $20^{\text {th }}$ century modern vaccine delivery platforms like recombinant viral vector vaccines, bacterial vector vaccines, DNA vaccines, Mrna vaccines, DNA vaccines, Outer membrane vesicles, virus like particles had a rapid growth, with many viral, bacterial vaccines being currently used and relatively newer vaccine technologies like Mrna vaccines, DNA vaccines, Virus like particles show a promising future with several vaccine candidates in development pipeline.
\end{abstract}

Keywords: Vaccines, mRNA Vaccine, DNA Vaccine, Small Pox, Recombinant DNA Technology

\section{Introduction}

Mankind has greatly benefited from vaccines for more than two centuries, yet the search for efficient vaccines is still a never-ending pursuit, The history of vaccines and vaccination starts with the first effort to prevent disease in the society. Smallpox was prevalent and is believed to have infested india and egypt over 3,000 years ago $[1,2]$. Observations about smallpox were made by physicians and scholars like Thucydides who reported that people affected by smallpox were immune from the future infections in 430 BC $[2,3]$. The evidence indicates that inoculation of smallpox material into sub-cutis was practiced in Indian subcontinent, ottoman empire and china by $1000 \mathrm{AD}[1,4,14]$.
Edward Jenner demonstrated immunity to smallpox in 1796, after he inoculated a 13-year-old-boy with vaccinia virus (cowpox), which made the boy immune to smallpox, "Jennerian inoculation" $[1,14]$ played an important role in effective eradication of small pox which plagued the world for several centuries

Vaccinology has been developing at a brisk pace since the advent of jennerian inoculation.

\section{Attenuation}

Live attenuated vaccine is produced by weakening the organism, decreasing its virulence, but still keeping it viable (or "live"). Live attenuated vaccines are derived from disease causing virus or 
bacteria in a laboratory The earliest of vaccines were developed on the idea of attenuation $[2,4]$.

\section{Inactivated Vaccine}

Consist of either whole viruses or bacteria that have been grown in culture and then killed/inactivated using physical (heat, or radiation) and chemical methods (usually formalin). The pathogen particles are destroyed and cannot replicate, but the antigens elicit an immune response [1]. Louis Pastuer discovered in 1881 that chickens injected with dead cultures of Pasteurella multocida did not contract disease when injected with live culture of Pasteurella multocida [1,2] Using similar principles he invented vaccines for rabies and anthrax. Inactivated Vaccines elicit a feebler immune response when compared to live attenuated vaccines but are safe for use in immuno-compromised individuals unlike live attenuated vaccines.

\section{Subunit vaccine}

Subunit vaccines contain only the antigenic parts of the pathogen necessary to elicit a protective immune response. Potential subunits are examined by simulating in order to study their prospective antigenic properties to speculate which particular combinations will produce an effective immune response Subunit vaccine can be produced by culturing the whole microbe and extracting desired antigen chemically or by recombinant DNA technology $[27,28]$.

Sub-unit vaccines are broadly classified as following

- $\quad$ Protein sub-unit vaccines

- Polysaccharide sub-unit vaccines

- Conjugate sub-unit vaccines

Subunit vaccines carry the advantage over live attenuated vaccines that they are safer to use in immune-compromised unlike live attenuated vaccines which pose a risk of causing infection in immuno-compromised individuals $[27,28]$.

Most of the vaccines that continue to be used today fall into the above-mentioned categories of vaccines the revolutions in genetic engineering in the second half had a great impact on vaccinology with introduction of recombinant dna technologies which opened up the possibility of using relatively harmless viruses/bacteria for delivering the antigens of disease causing agent thus completely eliminating the need for infectious agent to be introduced to the human/animal body for soliciting a immune response.
Viral vector vaccines

Non or low pathogenic viruses are selected. viral vectors are modified genetically by deleting genes coding for replication and replacing them by gene coding for target antigen. Most viral vectors are replication-defective and designed to deliver recombinant heterologous antigens to stimulate the host immune response [29].

MVA (Modified Vaccinia virus Ankara) vector and the Ad (Adenoviral) vector show promising results against HIV-1 in preliminary evaluation [29]. The CMV (Cytomegalo Virus) vector elicits a strong immune response, similarly the $\mathrm{SeV}$ (Sendai Virus) vector induces adequate mucosal immunity [29] making them both strong candidates as virus vectors for potential viral vector vaccines in the future.

Earliest clinical trials for a retroviral vector took place in last decade of $20^{\text {th }}$ century. But given the high probability of viral vector to combine with host genome which may cause genotoxicity, tumorigenesis have raised serious concerns about use of retroviral vectors as potential vectors [26]. The AAV (adeno associated virus) vectors express episomal genes without integrating with the host genome and thus have been approved by the European medical agency for clinical use in 2012 [26].

\section{Bacterial vector vaccines}

Genetically attenuated microorganisms, including pathogenic and commensal bacteria can be designed and modified to carry and deliver desired foreign antigens to elicit an immune response against both the pathogen from which the donor gene is derived $[7,17]$.

\section{Outer membrane vesicle vaccines}

Outer membrane vesicles (OMVs) exosomes that are released by gram negative bacteria during invivo/invitro growth. OMVs are bi-layered, spherical bacterial nanoparticles of size 25-250 $\mathrm{nm}$ [9].

OMVs possess the optimal size for recognition by immune cells, and present surface-exposed antigens of bacteria from which it is derived and Toll-like receptor (TLR) activating components, they induce both humoral and cell-mediated immune responses [15,21].

\section{Virus like particles}

Virus-like particles (VLP) are composed proteins from the viral capsid but lack any genomic material, precluding averting any possibility of reversal mutations or pathogenic infections [23]. 
VLPs cannot replicate in the vaccine recipient but stimulate the immune system through recognition of repetitive subunits [23], thus producing cellular and humoral immune responses of levels sufficient to protect the recipient againt the target virus VLPs are composed of one or several structural proteins that have the ability to self-assemble when recombinantly expressed [24].

VLPs can also have an external lipid envelope. In this case, the structural protein core exits the cell through a budding process, enveloping the capsid within part of the cell membrane [23,24].

Recently chimeric VLPs, have been developed. They posess structure of viral protein of one virus while the envelope proteins are derived from a second virus [24].

This opens the possibility of using VLPs as a delivery system. Envelope proteins can act as signals for specific tissue receptors. In this way, VLPs may be targeted to a given tissue, with capsid proteins modified to components to be delivered to the targeted tissue [24] making VLPs a promising venture given its potential applications in drug delivery, gene therapy, and cancer treatment.

The expression system and producer cell line for VLP production is chosen based on protein folding and post-translational modification requirement of VLPs [23,24].

Bacteria are suitable expression system for VLPs with just one or two structural proteins and no envelope [24].

Yeast cell lines have the ability to perform post-translational modifications making them suitable for production of VLPs that require post translational modifications [24].

Designing baculovirus genome is a easy, fast procedure making it suitable for the production of vaccines for fast mutating viruses whose surface protein structures can vary between each outbreak [24].

Mammalian cell lines can produce relatively simpler proteins but with complex and accurate post-translational modifications [24].

Transgenic plants can be used for VLP production. Agrobacterium tumefaciens is commonly used for infection and transformation of the cells These bacteria can infect plant cells and introduce a specific gene of interest into the host genome [24].
VLP based Hepatitis B vaccine is currently marketed by Glaxo smith and kline as Energix ${ }^{\mathrm{TM}}$ [23].

VLP based vaccines for Influenza A (H1N1), Hepatitis E, human papiloma virus, Human Immunodeficiency virus, Malaria is currently in development pipeline [23].

\section{DNA vaccines}

DNA vaccines comprise of circular piece of DNA (plasmid), which contains a foreign gene from a disease agent and a promoter that is used to initiate the expression of the protein from that gene in the target animal [25].

The recombinant plasmids containing a foreign gene is obtained from the bacterial culture by purification and the "naked" DNA is injected directly into the animal, usually intramuscularly or intradermally [10].

Transgenic plasmid reach antigen-presenting cells (APCs), like dendritic cells. The plasmids travel to the nucleus via endosomal trafficking, in nucleus the trans gene will be transcribed using the available nuclear transcription machinery plasmid remains episomal and transcriptionally active for several months. on mRNA reaching the endoplasmic reticulum, the antigen protein is synthesized. complete immune response involving MHC-I and MHC-II pathways Is triggered by DNA vaccines $[10,15]$.

First DNA Vaccine approved for use was west nile fever virus vaccine (West Nile-Innovator DNA ${ }^{\mathrm{TM}}$ ) in horses in 2005, DNA vaccines for haemorraghic disease of salmons are approved by European medical agency [25].

\section{RNA based vaccine}

Types of RNA vaccine

\section{Non-replicating mRNA}

The simplest type of RNA vaccine, an mRNA strand is packaged and delivered to the body, where it is taken up by the body's cells to make the antigen [16].

\section{In vivo self-replicating mRNA}

The pathogen-mRNA strand is packaged with additional RNA strands that ensure it will be copied once the vaccine is inside a cell. This means that greater quantities of the antigen are made from a smaller amount of vaccine, helping to ensure a more robust immune response $[8,16]$. 


\section{In vitro dendritic cell non-replicating mRNA vaccine}

Dendritic cells are immune cells that can present antigens on their cell surface to other types of immune cells to help stimulate an immune response. These cells are extracted from the patient's blood, transfected with the RNA vaccine, then given back to the patient to stimulate an immune reaction $[8,16]$.

\section{Self-amplifying mRNA vaccines}

Currently self-amplifying mRNA (SAM) vaccines are based on an alphavirus genome, where the genes encoding the RNA replication machinery are intact but the genes encoding the structural proteins are replaced with the antigen of interest $[8,16]$.

The SAM platform enables large amount of antigen production from an extremely small dose of vaccine $[16,20]$

An early study reported that immunization with $10 \mu \mathrm{g}$ of naked SAM vaccine encoding RSV fusion (F), influenza virus haemagglutinin (HA) or louping ill virus pre-membrane and envelope (prME) proteins resulted in antibody responses and partial protection from lethal viral challenges in mice [6].

\section{Dendritic cell mRNA vaccines}

ex vivo DC loading is a heavily pursued method to generate cell-mediated immunity against cancer. Development of infectious disease vaccines using this approach has been mainly limited to a therapeutic vaccine for HIV-1 [19].

Individuals on highly active antiretroviral therapy were treated with autologous DCs electroporated with mRNA encoding various HIV-1 antigens, and cellular immune responses were evaluated. This intervention proved to be safe and elicited antigen specific $\mathrm{CD}_{4+}$ and $\mathrm{CD}_{8+} \mathrm{T}$ cell responses but no clinical benefit was observed [26].

Another study in humans evaluated a CMV(Cytomegalovirus) pp65 mRNA-loaded DC vaccination in healthy human volunteers and allogeneic stem cell recipients and reported induction or expansion of CMV-specific cellular immune responses [19].

\section{Non-replicating mRNA vaccines}

RNA vaccine consists of an mRNA strand that codes for a disease-specific antigen [16].

Injected mRNA vaccine enters cells by cytosolic delivery and mRNA express antigen in target cell such as antigen-presenting cells (APCs) and elicit desired protective adaptive immune response for vaccines [16].

\section{Conclusion}

The rapid progress in bioengineering, computation has ushered a new era of vaccines, with a promising future where infectious, immune mediated diseases, diseases like cancer can be cures/prevented with vaccines.

Vaccine technologies like DNA vaccines, RNA vaccines and Virus like particles (VLP's) have shown promising results in pre-clinical testing and clinical trials,however this doesn't mean the end for conventional vaccines like attenuated, sub-unit and inactivated vaccines as it is highly unlikely that new vaccines will be developed for diseases where vaccines are available and work efficiently as vaccine research and development takes a serious toll on resources.

Newer, modern vaccines will be faster to develop and safer to use and will undoubtedly play a major role in handling of emerging, re-emerging diseases in future as witnessed in the awake of COVID-19 pandemic

\section{Bibliography}

1. Alexandra Minna Stern and Howard Marke. "The History of Vaccines and Immunization: Familiar Patterns New Challenges". Health Affairs 24.3 (2005).

2. Plotkin SA. "History of Vaccine Development". Springer, New York (2011).

3. Plotkin SL and Plotkin SA. "A short history of vaccination. Vaccines, eds Plotkin SA, Orenstein WA, Offit PA. (Elsevier-Saunders, Philadelphia), $6^{\text {th }}$ Ed (2013): 1-13.

4. Chandrakant Lahariya. "A brief history of vaccines and vaccination in India". Indian Journal of Medical Research 139 (2014): 491-511.

5. Norbert Pardi., et al. "mRNA vaccines - a new era in vaccinology" Nature Reviews. Drug Discovery 17 (2018): 261-279.

6. Linares-Ferna'ndez., et al. "Tailoring mRNA Vaccine to Balance Innate/Adaptive Immune Response". Trends in Molecular Medicine 26.3 (2019): 311-323.

7. Ivan YC Lin., et al. "Live-Attenuated Bacterial Vectors: Tools for Vaccine and Therapeutic Agent Delivery -Review Vaccines 3 (2015): 940-972. 
8. Nicholas AC Jackson., et al. "The promise of mRNA vaccines: a biotech and industrial perspective, review article". npj Vaccines 5 (2020): 1 .

9. Francesca Mancini., et al. "OMV Vaccines and the Role of TLR Agonists in Immune Response". International Journal of Molecular Sciences 21 (2020): 4416.

10. Michel Chartrain. "The production of plasmid Dna vaccine in Escherichia coli: a novel bacterial-based vaccine production platform". Vaccine Development and Manufacturing $1^{\text {st }}$ Edition.

11. Bernadette Ferraro., et al. "Clinical Applications of DNA Vaccines: Current Progress". Clinical Infectious Diseases 53.3 (2011): 296-302.

12. Mark W., et al. "Vaccine Development Using Recombinant DNA Technology". CAST (2008): 38.

13. Adilson José da Silva., et al. Brazilian Journal of Microbiology 45.4 (2014): 1117-1129.

14. Bhattacharya $S$ and Jackson M. "Vaccination against smallpox in India”. London: Welcome Institute" (2005).

15. Hajj KA., et al. "Branched-tail lipid nanoparticles potently deliver mRNA in vivo due to enhanced ionization at endosomal pH". Small 15 (2019): 1805097.

16. Pardi N., et al. "mRNA vaccines - a new era in vaccinology". Nature Reviews Drug Discovery 17 (2018): 261-279.

17. Shata MT., et al. "Recent advances with recombinant bacterial vaccine vectors". Molecular Medicine Today 6 (2000): 66-71.

18. Carleton HA. "Pathogenic bacteria as vaccine vectors: Teaching old bugs new tricks". Yale Journal of Biology and Medicine 83 (2010): 217-222.

19. John S., et al. "Multi-antigenic human cytomegalovirus mRNA vaccines that elicit potent humoral and cell mediated immunity". Vaccine 36 (2018): 1689-1699.

20. Ljungberg K and Liljestrom P. "Self-replicating alphavirus RNA vaccines”. Expert Review of Vaccines 14 (2015): 177-194.

21. Kaparakis-Liaskos M and Ferrero RL. "Immune modulation by bacterial outer membrane vesicles". Nature Reviews Immunology 15 (2015): 375-387.

22. Tan K., et al. “Outer Membrane Vesicles: Current Status and Future Direction of These Novel Vaccine Adjuvants". Frontiers in Microbiology 9 (2018): 783.
23. Liu Fuxiao., et al. "Virus-like particles: Potential veterinary vaccine immunogens". Research in veterinary science 93 (2011): 553-559.

24. Fuenmayor J., et al. "Production of virus-like particles for vaccines”. New Biotechnology 39 (2017): 174-180.

25. Gómez Leonardo and Oñate Angel. "Plasmid-Based DNA Vaccines (2018).

26. Gandhi Rajesh., et al. "Immunization of HIV-1-infected Persons with Autologous Dendritic Cells Transfected with mRNA Encoding HIV-1 Gag and Nef: Results of a Randomized, PlaceboControlled Clinical Trial". Journal of acquired immune deficiency syndromes 71.3 (1999): 246-253.

27. Douglas B Young. "Disease states and vaccines: selected cases: part h. Tuberculosis, Editor (s): Barry R. Bloom, Paul-Henri Lambert, The Vaccine Book, Academic Press (2003): 279-289.

28. KL Wilson., et al. "Inflammatory/Noninflammatory Adjuvants and Nanotechnology-The Secret to Vaccine Design". Editor (s): Mariusz Skwarczynski, Istvan Toth, In Micro and Nano Technologies, Micro and Nanotechnology in Vaccine Development, William Andrew Publishing (2017): 99-125.

29. Ura Takehiro., et al. "Developments in Viral Vector-Based Vaccines". Vaccines 2.3 (2014): 624-41.

\section{Assets from publication with us}

- Prompt Acknowledgement after receiving the article

- Thorough Double blinded peer review

- Rapid Publication

- Issue of Publication Certificate

- High visibility of your Published work

Website: www.actascientific.com/

Submit Article: www.actascientific.com/submission.php Email us: editor@actascientific.com

Contact us: +919182824667 